\title{
The role of fibrinogen in predicting reinfection after DAIR for periprosthetic joint infections
}

\author{
Dacheng Zhao ${ }^{\dagger}$, Jinwen $\mathrm{He}^{\dagger}$, Xingwen Wang, Xiaobing Zhao, Yayi Xia ${ }^{*}$ and Bin Geng
}

\begin{abstract}
Background: Fibrinogen (FIB) has been found to be a promising marker in diagnosing periprosthetic joint infection (PJI), however, the value of FIB in predicting reinfection of PJI is unknown. The purpose of this study was to evaluate the value of FIB in predicting reinfection after debridement, antibiotics, and implant retention (DAIR) for PJI.

Methods: We retrospectively analyzed the clinical data of patients who were diagnosed with PJI and underwent DAIR from 2013 to 2019. The levels of the FIB, erythrocyte sedimentation rate (ESR), and C-reactive protein (CRP) were measured before DAIR. After DAIR, patients were followed and reinfections were identified. For both acute and chronic PJI, the predictive value of FIB was evaluated by calculating the sensitivity, specificity, and area under the curve (AUC) of the receiver operating characteristic curve (ROC), and was compared with traditional inflammatory markers including ESR and CRP.
\end{abstract}

Results: The expression of FIB differed between patients reinfected and those not reinfected in both acute and chronic PJI $(p<0.05)$. In patients who underwent DAIR for acute PJl, the sensitivity and specificity of FIB were 81.82 and $83.33 \%$, respectively, which were significantly higher than that of CRP (sensitivity, $72.73 \%$; specificity, 50\%; $p<$ 0.05), while the specificity was higher than that of ESR (specificity, 41.67\%; $p<0.05$ ). In patients who underwent DAIR for chronic PJI, the sensitivity and specificity of FIB were 80.00 and $66.66 \%$, respectively, which were significantly higher than that of CRP (sensitivity, 53.33\%; specificity, 66.66\%; $p<0.05$ ) and ESR (sensitivity was 66.00\%; specificity, 16.66\%; $p<0.05$ ). The ROC curves showed that FIB demonstrated the highest AUC among the biomarkers in both acute and chronic PJI.

Conclusion: FIB is a promising indicator in predicting reinfection after DAIR for both acute and chronic PJI, and it seems to perform better than ESR and CRP.

Keywords: Fibrinogen, Periprosthetic joint infection, Debridement, Implant retention, Reinfection

\footnotetext{
* Correspondence: xiayy@|zu.edu.cn; ery_gengb@|zu.edu.cn

${ }^{\dagger}$ Dacheng Zhao and Jinwen He contributed equally to this work.

Department of Orthopaedics, Lanzhou University Second Hospital,

Orthopaedics Key Laboratory of Gansu Province, Orthopaedics Clinical

Research Center of Gansu Province, No. 82 of Cuiying Men, Lanzhou 730030,

Gansu, China
}

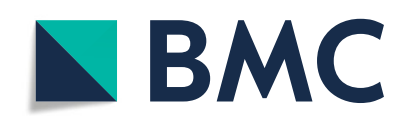

( The Author(s). 2021 Open Access This article is licensed under a Creative Commons Attribution 4.0 International License, which permits use, sharing, adaptation, distribution and reproduction in any medium or format, as long as you give appropriate credit to the original author(s) and the source, provide a link to the Creative Commons licence, and indicate if changes were made. The images or other third party material in this article are included in the article's Creative Commons licence, unless indicated otherwise in a credit line to the material. If material is not included in the article's Creative Commons licence and your intended use is not permitted by statutory regulation or exceeds the permitted use, you will need to obtain permission directly from the copyright holder. To view a copy of this licence, visit http://creativecommons.org/licenses/by/4.0/. The Creative Commons Public Domain Dedication waiver (http://creativecommons.org/publicdomain/zero/1.0/) applies to the data made available in this article, unless otherwise stated in a credit line to the data. 


\section{Introduction}

Periprosthetic joint infection (PJI) is a serious complication after primary hip and knee arthroplasty, and the primary reason for failure after surgery [1]. Treatment options for PJI include debridement, antibiotics, and implant retention (DAIR), 1-stage revision, 2-stage revision, joint fusion, and amputation [2-6]. The success rate of DAIR for PJI has been reported to be between 20 and $80 \%$ and related to obesity, comorbidities, operative time, perioperative period, microbial culture, and sensitivity to antibiotics [7-9].

Traditionally, diagnosis of infection after joint arthroplasty includes measuring serum levels of ESR and CRP [10-12], however, in the acute phase they can be influenced by the surgical intervention [13]. Furthermore, the formation of biofilms in the chronic stage may affect the utility of ESR and CRP to evaluate infection [14]. Therefore, new indicators are required for the assessment of acute and chronic PJI. FIB is a coagulation component that has been recently found to be an inflammatory marker useful in the diagnosis of PJI $[15,16]$. Our previous study showed that FIB was not inferior to ESR and CRP in differentiating PJI and aseptic loosening, and it was useful in assessing infection outcomes after first-stage surgery [14]. Serum D-dimer, also been reported for use in PJI diagnosis [17], is included in the new validated diagnostic criteria of PJI [18]. A recent multicenter study revealed that fibrinogen (FIB) performed better than D-dimer in PJI diagnosis, with similar sensitivity and specificity compared with ESR and CRP [19]. To our knowledge, whether FIB can be used to predict reinfection after DAIR is unknown. The purpose of this study was to evaluate the value of FIB in predicting reinfection in patients who underwent DAIR for PJI.

\section{Materials and methods \\ Patients}

We retrospectively reviewed the database of arthroplasty surgeries in our center from 2013 to 2019. Patients who were treated with DAIR for PJI after primary hip and knee arthroplasty were included. The definition of acute and chronic PJI followed the MSIS standard [20], in which the acute phase was defined as within 90 days and chronic phase was defined as more than 90 days after index arthroplasty. Patients who were diagnosed with acute hematogenous infections were not specified in our study cohort. The exclusion criteria considered factors that may influence the levels of biomarkers, including debridement or revision surgery previously, use of anticoagulant or antibiotics 2 weeks before admission, venous thromboembolism (VTE), autoimmune disease, hematologic diseases, liver diseases, and malignancies. The study was approved by the Ethics Committee of our hospital (2019A-211).

\section{Preoperative evaluation}

The 2018 criteria of the International Consensus Meeting (ICM) on musculoskeletal infection were used for the diagnosis of PJI [18]. After admission, the serum levels of ESR and CRP, and FIB were measured. An experiential antibiotic regimen of vancomycin (1 g, Q12h) and cefoperazone (3 g, Q8h) was used before the pathogen was identified. All patients underwent joint aspiration before DAIR with fluid sent for microbiologic examination. Blood and tissue samples were routinely cultured for 7 days with at least three specimens obtained. In culture-negative cases, tissue cultures were held for 14 days. Antibiotic regimens were tailored according to the identified pathogen and consultation from the pharmacy department. The interval between the index surgery and detection of infection and the identified pathogen were recorded. Methicillin-sensitive Staphylococcus aureus (MSSA) and methicillin-resistant Staphylococcus aureus (MRSA) were listed as subgroups of total Staphylococcus aureus isolates.

\section{DAIR and identification of reinfection}

All patients underwent standard DAIR, tissues obtained intraoperatively were sent for pathological and microbiologic examination, and the results were confirmed by experienced experts. Antibiotics were given intravenously for 2 weeks and orally for 4 weeks, and the regimens were adjusted according to the symptoms and laboratory examination. All patients were followed up for at least 1 year. The criteria for reinfection after DAIR were (1) Any reoperation for infection after surgery. (2) Long-term use of inhibitory antibiotics (more than 3 months). (3) Recurrent infections that were caused by strains of the same organism. (4) PJI related deaths.

\section{Statistical analysis}

The sample size required for this study was assessed by using PASS software (version 15) and the Power value asset 0.9 . Continuous variables were expressed as mean and standard deviation, and assessed by using t-test. The rank-sum test was used when the homogeneity of variance of continuous variables was not equal. The predictive values of FIB, ESR, and CRP were evaluated by calculating the sensitivity, specificity, positive predictive value (PPV), and negative predictive value (NPV), and area under the curve (AUC) in the receiver operating characteristic (ROC) curve. AUC was defined as the area under the ROC 
curve and the coordinate axis, the discriminative power was defined as outstanding (0.90-1.00), good (0.80-0.89), general (0.70-0.79), the difference $(0.60-$ $0.69)$, or unqualified (0.50-0.59). The optimal cutoff was determined by calculating the Youden index. Comparison between variables was run by using the chi-square test. All statistical analyses were performed by using IBM SPSS statistical software (Version 25), MedCalc statistical software (version 19). The significance value was defined as 0.05 .

\section{Results}

From January 2013 to August 2019, 44 patients were included for analysis. Twenty-three patients were diagnosed with acute PJI (acute PJI group) and 21 patients were diagnosed with chronic PJI (chronic PJI group). There were no significant differences in demographic data including age, sex, body mass index (BMI), and involved joints between the two groups $(p>0.05$, Tables 1 and 2). Patients were furtherly divided into subgroups according to whether they developed reinfection after DAIR. Eleven patients $(47.8 \%)$ in the acute PJI group and 15 patients $(71.4 \%)$ in the chronic PJI group were diagnosed with reinfection after DAIR. There was no significant difference in reinfection rate between the acute and chronic PJI groups $(p=0.136)$.

\section{Diagnose of PJI and pathogens}

In the acute PJI group, the interval between index surgery and identification of PJI was 1.50 (1.10, 2.10) months in the reinfection patients and $1.25(0.925,1.95)$ months in the non-reinfection patients $(p=0.347)$. In the chronic PJI group, the interval between index surgery and identification of PJI was 11.50 (7.9-21.20) months in the reinfection patients and 12.50 (4.1526.50) months in the non-reinfection patients ( $p=$ 0.622). The interval between index surgery and

Table 1 Demographic information of patients with acute PJI treated with DAIR

\begin{tabular}{llll}
\hline Characteristic & \multicolumn{2}{l}{ Reinfection } & P value \\
\cline { 2 - 3 } & Yes $(\boldsymbol{n}=\mathbf{1 1})$ & No $(\boldsymbol{n}=\mathbf{1 2})$ & \\
\hline Age $(\mathrm{y})$ & $63.18 \pm 8.62$ & $63.75 \pm 11.89$ & 0.898 \\
Gender & & & 0.912 \\
$\quad$ Male & $2(18.2 \%)$ & $3(25.0 \%)$ & \\
Female & $9(81.8 \%)$ & $9(75.0 \%)$ & 0.499 \\
BMl (kg/m2) & $25.32 \pm 4.88$ & $26.61 \pm 4.06$ & 0.296 \\
Involved joint & & & \\
Knee & $6(54.5 \%)$ & $10(83.3 \%)$ & \\
Hip & $5(45.5 \%)$ & $2(16.7 \%)$ & \\
\hline All quantita & & & \\
\hline
\end{tabular}

All quantitative data were expressed as mean \pm standard deviation $P J I$ Periprosthetic joint infection, DAIR Debridement, antibiotics, and implant retention, $B M I$ Body mass index
Table 2 Demographic information with chronic PJI treated with DAIR

\begin{tabular}{llll}
\hline Characteristic & \multicolumn{2}{l}{ Reinfection } & P value \\
\cline { 2 - 3 } & Yes $(\boldsymbol{n}=\mathbf{1 5})$ & No $(\boldsymbol{n}=\mathbf{6})$ & \\
\hline Age (y) & $64.27 \pm 9.19$ & $64.50 \pm 3.51$ & 0.934 \\
Gender & & & 0.944 \\
$\quad$ Male & $9(18.2 \%)$ & $350.0 \%)$ & \\
$\quad$ Female & $6(81.8 \%)$ & $3(50.0 \%)$ & \\
BMl (kg/m2) & $24.85 \pm 3.33$ & $25.34 \pm 2.39$ & 0.749 \\
Involved joint & & & 0.831 \\
$\quad$ Knee & $9(54.5 \%)$ & $4(66.7 \%)$ & \\
$\quad$ Hip & $6(45.5 \%)$ & $2(33.3 \%)$ & \\
\hline
\end{tabular}

All quantitative data were expressed as mean \pm standard deviation $P J I$ Periprosthetic joint infection, DAIR Debridement, antibiotics, and implant retention, $B M I$ body mass index

identification of PJI and the identified pathogens in each subgroup were shown in Tables 3 and 4 .

\section{Predictive value of biomarkers}

In both acute and chronic PJI groups, there were no significant differences in the levels of ESR and CRP between the reinfection and no reinfection patients ( $p>0.05$, Tables 3 and 4 ), however, the levels of FIB in the reinfection group were significantly higher than that of the no reinfection group $(p<0.05$, Tables 3 and 4 ).

In the acute PJI group, the ROC curves showed that FIB demonstrated the highest AUC of 0.75, with an optimal cutoff of 3.32. The sensitivity, specificity, PPV, NPV were $81.82,83.33,81.80,83.33 \%$, respectively. ESR showed an AUC of 0.63 , the cutoff point was 22, the sensitivity, specificity, PPV, NPV were 90.91, 41.67, 58.80, 83.30\%, respectively. CRP demonstrated an AUC of 0.56 , with a cutoff point of 58.62 , the sensitivity, specificity, PPV, NPV were 72.73, 50.00, 57.10, 66.70\%, respectively (Fig. 1; Table 5).

In the chronic PJI group, the ROC curves showed that FIB demonstrated the highest AUC of 0.81 , with an optimal cutoff of 3.94. The sensitivity, specificity, PPV, NPV were $80.00,66.66,85.71,57.14 \%$, respectively. ESR showed an AUC of 0.56, the cutoff point was 20, the sensitivity, specificity, PPV, NPV were 66.00, 16.66, 66.66, 16.66\%, respectively. CRP demonstrated an AUC of 0.58 , with a cutoff point of 16.50 , the sensitivity, specificity, PPV, NPV were 53.00, 66.66, 80.00,36.36\%, respectively (Fig. 2; Table 5).

In summary, the AUC of FIB was highest among the three biomarkers. The sensitivity, specificity, PPV, and NPV of FIB were higher than that of ESR and CRP in the chronic PJI group. Similar results were observed in the acute PJI group except for sensitivity, FIB had a lower sensitivity than ESR. 
Table 3 Biomarker levels before DAIR in patients with acute PJI

\begin{tabular}{|c|c|c|c|}
\hline \multirow[t]{2}{*}{ Items } & \multicolumn{2}{|l|}{ Reinfection } & \multirow[t]{2}{*}{$P$ value } \\
\hline & Yes $(n=11)$ & No $(n=12)$ & \\
\hline $\operatorname{ESR}(\mathrm{mm} / \mathrm{h})$ & $42.00(38.00,65.00)$ & $39.00(20.25,49.50)$ & 0.288 \\
\hline $\mathrm{CRP}(\mathrm{mg} / \mathrm{L})$ & $48.60(27.54,88.50)$ & $61.44(38.93,103.12)$ & 0.651 \\
\hline $\mathrm{FIB}(\mathrm{g} / \mathrm{L})$ & $4.03(3.45,4.61)$ & $3.08(2.92,3.32)$ & $<0.05$ \\
\hline Interval between index surgery and PJI (mon) & $1.50(1.10,2.10)$ & $1.25(0.925,1.95)$ & 0.347 \\
\hline \multicolumn{4}{|l|}{ Culture } \\
\hline Staphylococcus epidermidis & $3(27.3 \%)$ & $2(16.7 \%)$ & \\
\hline Hemolytic streptococcus & $2(18.2 \%)$ & $2(16.7 \%)$ & \\
\hline Total Staphylococcus aureus isolates & $6(54.5 \%)$ & $8(66.6 \%)$ & \\
\hline
\end{tabular}

The data were expressed as median (interquartile range)

$P J I$ Periprosthetic joint infection, DAIR Debridement, antibiotics, and implant retention, ESR Erythrocyte sedimentation rate, CRP C-reactive protein, FIB Fibrinogen, MSSA Methicillin-sensitive Staphylococcus aureus

\section{Discussion}

In the present study, we found that FIB was a promising biomarker in predicting reinfection after DAIR for both acute and chronic PJI, and it seemed to perform better than ESR and CRP. We reviewed the literature and confirmed that this is the first study assessing the predictive value of FIB after DAIR for acute and chronic PJI.

Management of PJI is challenging. DAIR is recommended for acute PJI by the International Consensus [21], as it avoids reimplanting the prosthesis and can decrease the risk of complications. DAIR for chronic PJI is also reported [22]. Since the failure of DAIR is common in clinical practice, especially for chronic PJIs, the identification of those at increased risk for reinfection after DAIR is crucial.
The detection of reinfection after DAIR has been previously reported. Maier et al. [23] found that ESR had a sensitivity of $58 \%$, and a specificity of $66 \%$ for reinfection after DAIR performed for acute postoperative infection; while CRP showed a sensitivity of $42 \%$, and specificity of 56\%. For chronic infection, ESR showed a sensitivity of $67 \%$, and a specificity of $47 \%$, while CRP showed a sensitivity of $50 \%$ and a specificity of $26 \%$. Kuiper et al. [24] found that the failure rate of DAIR increased when ESR was higher than 60 $\mathrm{mm} / \mathrm{h}$. Similarly, a multicenter retrospective study showed that a high CRP level was associated with failure after DAIR [8]. Though ESR and CRP are traditional inflammatory indicators that are widely used to evaluate postoperative infections [12, 25, 26], they can be influenced by surgical intervention.

Table 4 Biomarker levels before DAIR in patients with chronic PJI

\begin{tabular}{|c|c|c|c|}
\hline \multirow[t]{2}{*}{ Items } & \multicolumn{2}{|l|}{ Reinfection } & \multirow[t]{2}{*}{$P$ value } \\
\hline & Yes $(n=15)$ & No $(n=6)$ & \\
\hline $\mathrm{ESR}(\mathrm{mm} / \mathrm{h})$ & $34.00(17.00,68.00)$ & $35.50(20.00,72.50)$ & 0.733 \\
\hline $\mathrm{CRP}(\mathrm{mg} / \mathrm{L})$ & $23.26(5.62,33.98)$ & $13.06(4.02,32.22)$ & 0.622 \\
\hline $\mathrm{FIB}(\mathrm{g} / \mathrm{L})$ & $4.28(3.98,5.12)$ & $3.68(3.20,4.05)$ & $<0.05$ \\
\hline Interval between index surgery and PJI (mon) & $11.50(7.9,21.20)$ & $12.50(4.15,26.50)$ & 0.622 \\
\hline \multicolumn{4}{|l|}{ Culture } \\
\hline Staphylococcus epidermidis & $4(26.7 \%)$ & $1(16.7 \%)$ & \\
\hline Hemolytic streptococcus & $2(13.3 \%)$ & $2(33.3 \%)$ & \\
\hline Negative & $3(20.0 \%)$ & $1(16.7 \%)$ & \\
\hline Escherichia coli & $2(13.3 \%)$ & 0 & \\
\hline Total Staphylococcus aureus isolates & $4(26.7 \%)$ & $2(33.3 \%)$ & \\
\hline MSSA & $2(13.3 \%)$ & $2(33.3 \%)$ & \\
\hline MRSA & $2(13.3 \%)$ & 0 & \\
\hline
\end{tabular}

The data were expressed as median (interquartile range)

$P J /$ Periprosthetic joint infection, DAIR Debridement, antibiotics, and implant retention, ESR Erythrocyte sedimentation rate, CRP C-reactive protein, FIB Fibrinogen, MSSA Methicillin-sensitive Staphylococcus aureus, MRSA Methicillin-resistant Staphylococcus aureus 


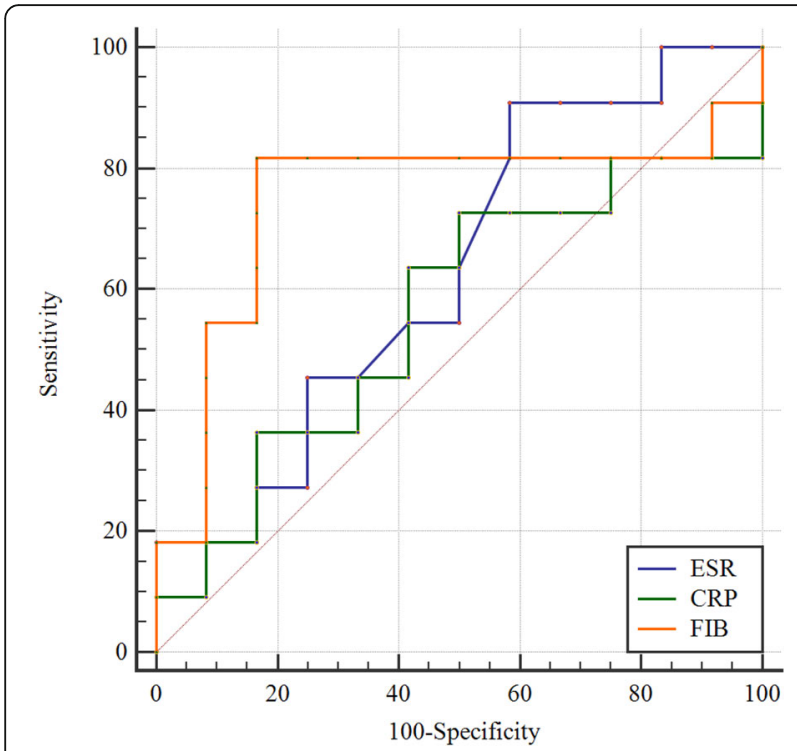

Fig. 1 Receiver operating characteristic curve analysis for predicting reinfection after DAIR treatment for acute periprosthetic joint infection. DAIR debridement, antibiotics, and implant retention, ESR erythrocyte sedimentation rate, CRP C-reactive protein, FIB fibrinogen

Previous studies found that the value of a single biomarker is limited in diagnosing PJI. Researchers attempted to combine single biomarkers into a multibiomarker model to improve diagnostic properties, and found that CRP and FIB performed best amongst all biomarkers. However, the construction of a multibiomarker model was not feasible [27].

The present study demonstrated 47.8 and $71.43 \%$ reinfection rates after DAIR for acute and chronic PJI respectively, which is similar to previous studies [28]. With regard to ESR and CRP in predicting failure, our results of specificity in the acute group and sensitivity in the chronic group were similar to that of Maier et al. [23], while sensitivity in the acute group and specificity in the chronic group were higher than their findings. Additionally, we showed a higher AUC for ESR and CRP, in both the acute and chronic PJI patients.

We found that in comparison to CRP, FIB had higher sensitivity and specificity in both acute and chronic reinfections. In comparison to ESR, FIB had higher specificity in acute reinfection and higher sensitivity and specificity in chronic reinfection. Since data of FIB in predicting reinfection after DAIR is lacking, the comparison of FIB with other studies was not available. However, our results revealed that FIB had a better discriminative power in predicting reinfection, indicating that FIB appears to be a screening indicator of reinfection for patients undergoing DAIR for both acute and chronic PJI. The ideal biomarker for any condition should be reliable and
Table 5 Diagnostic value of biomarkers for acute and chronic PJI treated with DAIR

\begin{tabular}{lll}
\hline $\begin{array}{l}\text { Values of } \\
\text { biomarkers }\end{array}$ & $\begin{array}{l}\text { Acute PJI }(\boldsymbol{n}=\mathbf{2 3}), \\
\text { Reinfection }(\boldsymbol{n}=\mathbf{1 1})\end{array}$ & $\begin{array}{l}\text { Chronic PJI }(\boldsymbol{n}=\mathbf{2 1}) \\
\text { Reinfection }(\boldsymbol{n}=\mathbf{1 5})\end{array}$ \\
\hline ESR & & \\
AUC & 0.63 & 0.56 \\
Optimal cutoff & $22 \mathrm{~mm} / \mathrm{h}$ & $20 \mathrm{~mm} / \mathrm{h}$ \\
Youden index & 0.33 & 0.23 \\
Sensitivity & $90.91 \%$ & $66.00 \%$ \\
Specificity & $41.67 \%$ & $16.66 \%$ \\
PPV & $58.80 \%$ & $66.66 \%$ \\
NPV & $83.30 \%$ & $16.66 \%$ \\
CRP & & \\
AUC & 0.56 & 0.58 \\
Optimal cutoff & $58.62 \mathrm{mg} / \mathrm{L}$ & $16.50 \mathrm{mg} / \mathrm{L}$ \\
Youden index & 0.23 & 0.37 \\
Sensitivity & $72.73 \%$ & $53.33 \%$ \\
Specificity & $50.00 \%$ & $66.66 \%$ \\
PPV & $57.10 \%$ & $80.00 \%$ \\
NPV & $66.70 \%$ & $36.36 \%$ \\
FIB & & \\
AUC & $81.76 \%$ & 0.81 \\
Optimal cutoff & $3.32 \mathrm{~g} / \mathrm{L}$ & $3.94 \mathrm{~g} / \mathrm{L}$ \\
Youden index & 0.65 & 0.63 \\
Sensitivity & $81.82 \%$ & $80.00 \%$ \\
Specificity & $83.33 \%$ & $57.14 \%$ \\
PPV & $83.30 \%$ & \\
NPV & & \\
\hline
\end{tabular}

ROC Receiver operating characteristic, ESR Erythrocyte sedimentation rate, CRP C-reactive protein, FIB Fibrinogen, AUC Area under the curve, PPV Positive predictive value, NPV The negative predictive value

reproducible, sensitive and specific, and provide risk stratification. Since FIB is a component of the coagulation test and is originally performed after admission, it is convenient and cost-effective compared to other inflammatory biomarkers.

The application of FIB does have several limitations, including that levels could be influenced by anticoagulant use, and that patients with thromboembolic disease, autoimmune disease, liver disease, among other conditions, were excluded in this study. As a result, how applicable FIB would be in the real world remains to be seen. Additionally, the study design was retrospective with a small sample size and interpretation may be influenced by selection bias and the composition of the study cohort. Furthermore, outcomes in PJI could be influenced by drug sensitivity, antibiotic regimen, comorbidities, and duration of the infection $[29,30]$. Furthermore, it was difficult to design a well-matched comparison group. Large, randomized controlled prospective studies are necessary to further confirm our findings. 


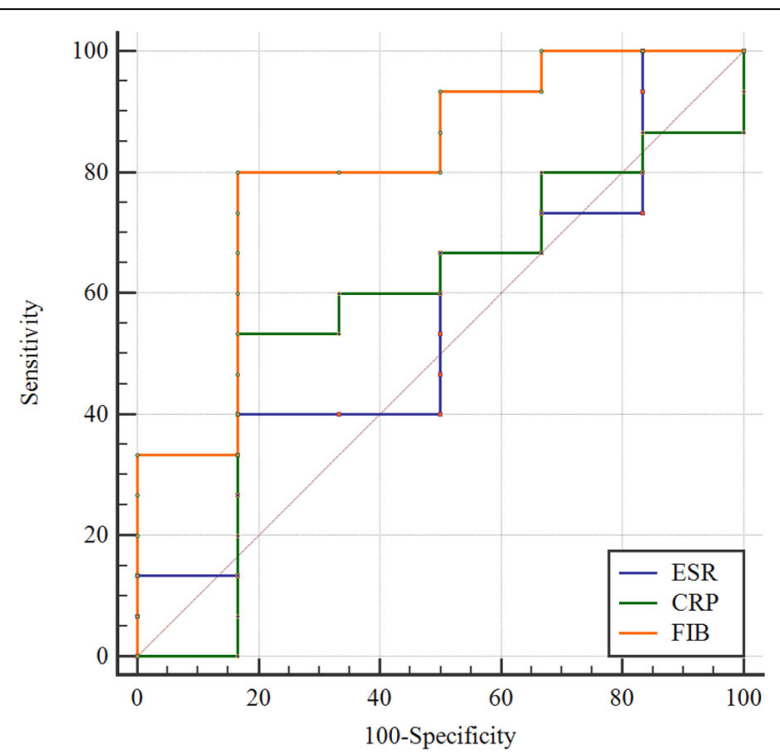

Fig. 2 Receiver operating characteristic curve analysis for predicting reinfection after DAIR treatment for chronic periprosthetic joint infection. DAIR debridement, antibiotics, and implant retention, ESR erythrocyte sedimentation rate, CRP C-reactive protein, FIB fibrinogen

\section{Conclusion}

FIB is a promising indicator in predicting reinfection after DAIR for both acute and chronic PJI, and it seems to perform better than ESR and CRP.

\section{Abbreviations}

CRP: C-reactive protein; ESR: Erythrocyte sedimentation rate; FIB: Fibrinogen; MSIS: Musculoskeletal Infection Society; PJl: Periprosthetic joint infection; DAIR: Debridement, antibiotics, and implant retention; WBC: White blood cell; ROC: Receiver operating Characteristic curve; AUC: Area under the curve; PPV: Positive predictive value; NPV: Negative predictive value; VTE: Venous thromboembolism

\section{Supplementary Information}

The online version contains supplementary material available at https://doi. org/10.1186/s12891-021-04357-8

\section{Additional file 1.}

\section{Acknowledgments}

None.

\section{Authors' contributions}

Yayi Xia and Bin Geng contributed to the study conception and design. Material preparation, data collection, and analysis were performed by Dacheng Zhao, Jinwen He, Xingwen Wang, and Xiaobing Zhao. The first draft of the manuscript was written by Dacheng Zhao and all authors commented on previous versions of the manuscript. All authors read and approved the final manuscript.

\section{Funding}

This study was supported by The National Natural Science Foundation of China (81874017, 81960403, and 82060405); The Natural Science Foundation of Gansu Province of China (20JR5RA320); Cuiying Scientific and Technological Innovation Program of Lanzhou University Second Hospital
(CY2017-ZD02); Science and Technology Plan Project of Lanzhou (2016-3121).

\section{Availability of data and materials}

All data generated or analyzed during this study are included in this published article [and its supplementary information files].

\section{Declarations}

Ethics approval and consent to participate

This study was conducted in accordance with the ethical guidelines of the Declaration of Helsinki. This retrospective study was approved by the Research Ethics Committee of the Second Hospital of Lanzhou University (2019A-211). Informed consent was obtained from all patients for this study, all patients agreed to participate in the study.

\section{Consent for publication}

All patients agreed to have their data published.

\section{Competing interests}

The authors declare that there is no conflict of competing financial and nonfinancial interests.

Received: 25 January 2021 Accepted: 10 May 2021

Published online: 24 May 2021

References

1. Parvizi J, Shohat N, Gehrke T. Prevention of periprosthetic joint infection: new guidelines. Bone Joint J. 2017;99-b(4 Supple B):3-10.

2. Achermann Y, Stasch P, Preiss S, Lucke K, Vogt M. Characteristics and treatment outcomes of 69 cases with early prosthetic joint infections of the hip and knee. Infection. 2014;42(3):511-9. https://doi.org/10.1007/s15010014-0584-6.

3. Di Benedetto P, Di Benedetto ED, Salviato D, Beltrame A, Gissoni R, Cainero $V$, et al. Acute periprosthetic knee infection: is there still a role for DAIR? Acta Biomed. 2017;88(2s):84-91. https://doi.org/10.23750/abm.v88i2-S.6518.

4. Sherrell JC, Fehring TK, Odum S, Hansen E, Zmistowski B, Dennos A, et al. The Chitranjan Ranawat Award: fate of two-stage reimplantation after failed irrigation and débridement for periprosthetic knee infection. Clin Orthop Relat Res. 2011;469(1):18-25. https://doi.org/10.1007/s1 1999-010-1434-1.

5. Tan TL, Goswami K, Fillingham YA, Shohat N, Rondon AJ, Parvizi J. Defining treatment success after 2-stage exchange arthroplasty for periprosthetic joint infection. J Arthroplast. 2018;33(11):3541-6. https://doi.org/10.1016/j.a rth.2018.06.015

6. Kuo FC, Goswami K, Shohat N, Blevins K, Rondon AJ, Parvizi J. Two-stage exchange arthroplasty is a favorable treatment option upon diagnosis of a fungal periprosthetic joint infection. J Arthroplast. 2018;33(11):3555-60. https://doi.org/10.1016/j.arth.2018.07.024.

7. Löwik CM, Parvizi J, Jutte PC, Zijlstra WP, Knobben BaS XC, et al. Debridement, antibiotics, and implant retention is a viable treatment option for early periprosthetic joint infection presenting more than 4 weeks after index arthroplasty. Clin Infect Dis. 2020;71(3):630-6. https://doi.org/10.1093/ $\mathrm{cid} / \mathrm{ciz} 867$.

8. Lora-Tamayo J, Murillo O, Iribarren JA, Soriano A, Sánchez-Somolinos M, Baraia-Etxaburu JM, et al. A large multicenter study of methicillin-susceptible and methicillin-resistant Staphylococcus aureus prosthetic joint infections managed with implant retention. Clin Infect Dis. 2013;56(2):182-94. https:// doi.org/10.1093/cid/cis746.

9. Rodríguez-Pardo D, Pigrau C, Lora-Tamayo J, Soriano A, Del Toro MD, Cobo $J$, et al. Gram-negative prosthetic joint infection: outcome of a debridement, antibiotics and implant retention approach. A large multicentre study. Clin Microbiol Infect. 2014;20(11):0911-9. https://doi.org/10.1111/1469-0691.1264 9.

10. Fujimoto $T$, Kaneko T, Sunakawa T, Ikegami H, Musha Y. Elevation of fibrin degradation product (FDP) values prevents the negative conversion of serum CRP values after total knee arthroplasty. J Orthop. 2018;15(4):940-4. https://doi.org/10.1016/j.jor.2018.08.005.

11. Stambough JB, Curtin BM, Odum SM, Cross MB, Martin JR, Fehring TK. Does change in ESR and CRP guide the timing of two-stage arthroplasty reimplantation? Clin Orthop Relat Res. 2019:477(2):364-71. https://doi.org/1 0.1097/01.blo.0000533618.31937.45. 
12. Schiffner E, Latz D, Thelen S, Grassmann JP, Karbowski A, Windolf J, et al. Normal CRP and WBC values in total hip arthroplasty (THA) with signs of loosening. Do we need a joint aspiration? J Clin Orthop Trauma. 2019;10(3): 566-70. https://doi.org/10.1016/j.jcot.2018.09.011.

13. Parvizi J, Gehrke T. Definition of periprosthetic joint infection. J Arthroplast. 2014:29(7):1331. https://doi.org/10.1016/j.arth.2014.03.009.

14. Bin $G$, Xinxin $Y$, Fan $L$, Shenghong $W$, Yayi $X$. Serum fibrinogen test performs well for the diagnosis of periprosthetic joint infection. J Arthroplast. 2020;35(9):2607-12. https://doi.org/10.1016/j.arth.2020.04.081.

15. Klim SM, Amerstorfer F, Gruber G, Bernhardt GA, Radl R, Leitner L, et al. Fibrinogen - a practical and cost efficient biomarker for detecting periprosthetic joint infection. Sci Rep. 2018;8(1):8802. https://doi.org/10.103 8/s41598-018-27198-3.

16. Zhang Q, Dong J, Zhou D, Liu F. Circulating D-dimer versus fibrinogen in the diagnosis of peri-prosthetic joint infection: a meta-analysis. Surg Infect. 2021;22(2):200-10. https://doi.org/10.1089/sur.2019.298.

17. Shahi A, Kheir MM, Tarabichi M, Hosseinzadeh HRS, Tan TL, Parvizi J. Serum D-dimer test is promising for the diagnosis of periprosthetic joint infection and timing of reimplantation. J Bone Joint Surg Am. 2017;99(17):1419-27. https://doi.org/10.2106/JBJS.16.01395.

18. Parvizi J, Tan TL, Goswami K, Hiquera C, Della Valle C, Chen AF, et al. The 2018 definition of periprosthetic hip and knee infection: an evidence-based and validated criteria. J Arthroplasty. 2018;33(5):1309-14.e2

19. Li R, Shao HY, Hao LB, Yu BZ, Qu PF, Zhou YX, et al. Plasma fibrinogen exhibits better performance than plasma D-dimer in the diagnosis of periprosthetic joint infection: a multicenter retrospective study. J Bone Joint Surg Am. 2019;101(7):613-9. https://doi.org/10.2106/JBJS.18.00624.

20. Parvizi J, Zmistowski B, Berbari EF, Bauer TW, Springer BD, Della Valle CJ, et al. New definition for periprosthetic joint infection: from the workgroup of the musculoskeletal infection society. Clin Orthop Relat Res. 2011;469(11): 2992-4. https://doi.org/10.1007/s11999-011-2102-9.

21. Argenson JN, Arndt M, Babis G, Battenberg A, Budhiparama N, Catani F, et al. Hip and knee section, treatment, debridement and retention of implant: proceedings of international consensus on orthopedic infections. J Arthroplast. 2019;34(2s):S399-s419. https://doi.org/10.1016/j.arth.2018.09.025.

22. Gramlich Y, Johnson T, Kemmerer M, Walter G, Hoffmann R, Klug A. Salvage procedure for chronic periprosthetic knee infection: the application of DAIR results in better remission rates and infection-free survivorship when used with topical degradable calcium-based antibiotics. Knee Surg Sports Traumatol Arthrosc. 2020;28(9):2823-34. https://doi.org/10.1007/s00167-01905627-8.

23. Maier SP, Klemt C, Tirumala V, Oganesyan R, Van Den Kieboom J, Kwon YM. Elevated ESR/CRP ratio is associated with reinfection after debridement, antibiotics, and implant retention in chronic periprosthetic joint infections. $J$ Arthroplast. 2020;35(11):3254-60. https://doi.org/10.1016/j.arth.2020.06.007.

24. Kuiper JW, Vos SJ, Saouti R, Vergroesen DA, Graat HC, Debets-Ossenkopp YJ, et al. Prosthetic joint-associated infections treated with DAIR (debridement, antibiotics, irrigation, and retention): analysis of risk factors and local antibiotic carriers in 91 patients. Acta Orthop. 2013:84(4):380-6. https://doi. org/10.3109/17453674.2013.823589.

25. Ishii Y, Noguchi H, Takeda M, Sato J, Takayama S, Toyabe S. Characteristics and significance of fever during 4 weeks after primary total knee arthroplasty. Arch Orthop Trauma Surg. 2014;134(5):707-12. https://doi.org/1 0.1007/s00402-014-1949-0.

26. Dupont C, Rodenbach J, Flachaire E. The value of C-reactive protein for postoperative monitoring of lower limb arthroplasty. Ann Readapt Med Phys. 2008;51(5):348-57. https://doi.org/10.1016/j.annrmp.2008.01.014.

27. Klim SM, Amerstorfer F, Glehr G, Hauer G, Smolle MA, Leitner L, et al. Combined serum biomarker analysis shows no benefit in the diagnosis of periprosthetic joint infection. Int Orthop. 2020;44(12):2515-20. https://doi. org/10.1007/s00264-020-04731-6.

28. Koyonos L, Zmistowski B, Della Valle CJ, Parvizi J. Infection control rate of irrigation and débridement for periprosthetic joint infection. Clin Orthop Relat Res. 2011;469(11):3043-8. https://doi.org/10.1007/s11999-011-1910-2.

29. Tornero E, Martínez-Pastor JC, Bori G, García-Ramiro S, Morata L, Bosch J, et al. Risk factors for failure in early prosthetic joint infection treated with debridement. Influence of etiology and antibiotic treatment. J Appl Biomater Funct Mater. 2014;12(3):129-34. https://doi.org/10.5301/jabfm. 5000209.

30. Bedair HS, Katakam A, Bedeir YH, Yeroushalmi D, Schwarzkopf R. A decision analysis of treatment strategies for acute periprosthetic joint infection: early irrigation and debridement versus delayed treatment based on organism. J Orthop. 2020;22:246-50.

\section{Publisher's Note}

Springer Nature remains neutral with regard to jurisdictional claims in published maps and institutional affiliations.

\section{Ready to submit your research? Choose BMC and benefit from:}

- fast, convenient online submission

- thorough peer review by experienced researchers in your field

- rapid publication on acceptance

- support for research data, including large and complex data types

- gold Open Access which fosters wider collaboration and increased citations

- maximum visibility for your research: over $100 \mathrm{M}$ website views per year

At BMC, research is always in progress.

Learn more biomedcentral.com/submissions 